\title{
How Can We Predict a Successful Outcome after Varicocelectomy in Painful Varicocele Patients? An Updated Meta-Analysis
}

\author{
Jih Hoon Park ${ }^{1,3}$ (D) Kyoungjune Pak ${ }^{2,3}$ (i) , Nam Cheol Park ${ }^{1,3}$ (i), Hyun Jun Park ${ }^{1,3}$ (iD \\ Departments of ${ }^{1}$ Urology and ${ }^{2}$ Nuclear Medicine, Pusan National University School of Medicine, ${ }^{3}$ Pusan National University Hospital \\ Biomedical Research Institute, Busan, Korea
}

\begin{abstract}
Purpose: This study evaluated the relationships of varicocele characteristics and surgical methods with the pain resolution rate post-varicocelectomy.

Materials and Methods: A literature search was conducted using PubMed (MEDLINE) and Embase (1980 to December 2018). The keywords used for the search were "varicocele," "varicocelectomy OR ligation OR repair," and "pain OR painful." Two authors independently reviewed the titles and abstracts of the retrieved articles. Studies were evaluated for eligibility, and those that fulfilled all of the inclusion criteria and none of the exclusion criteria were selected for full-text review.

Results: Fourteen studies were included in the analysis. There were no significant differences in the postoperative pain resolution rate with respect to varicocele grades I, II, and III. The resolution rate was significantly higher for dull pain than for dragging and sharp pain (odds ratio [OR], 5.73; 95\% confidence interval $[\mathrm{Cl}], 1.78-18.41 ; \mathrm{p}=0.003$ and $\mathrm{OR}, 8.72 ; 95 \% \mathrm{Cl}$, 2.91-26.08; $p=0.0001$, respectively). In terms of the surgical approach, subinguinal ligation was significantly more effective in terms of pain resolution than high ligation $(\mathrm{OR}, 2.73 ; 95 \% \mathrm{Cl}, 1.54-4.85 ; \mathrm{p}=0.0006)$. Microsurgical varicocelectomy showed a significantly higher likelihood of pain resolution than laparoscopic varicocelectomy $(\mathrm{OR}, 7.12 ; 95 \% \mathrm{Cl}, 3.78-13.44$; $\mathrm{p}<0.0001$ ).

Conclusions: Patients with varicocele complaining of dull pain preoperatively had a higher likelihood of postoperative pain resolution than those complaining of dragging or sharp pain. Furthermore, subinguinal ligation was more effective for pain resolution than high ligation, and microsurgical varicocelectomy showed better surgical outcomes than laparoscopic varicocelectomy.
\end{abstract}

Keywords: Meta-analysis; Pain; Varicocele; Varicocele repair; Varicocelectomy

This is an Open Access article distributed under the terms of the Creative Commons Attribution Non-Commercial License (http://creativecommons.org/licenses/by-nc/4.0) which permits unrestricted non-commercial use, distribution, and reproduction in any medium, provided the original work is properly cited.

\section{INTRODUCTION}

Chronic scrotal pain is defined as chronic or intermittent scrotal pain of at least 3 months, with various levels of severity, that interferes with daily activities and prompts the patient to seek medical treatment [1]. Common causes of scrotal pain include varicocele, epididymitis, spermatocele, tumors, infection, and torsion.

Received: Aug 18, 2019 Revised: Oct 17, 2019 Accepted: Oct 22, 2019 Published online Dec 3, 2019

Correspondence to: Hyun Jun Park (iD https://orcid.org/0000-0003-0566-9574

Department of Urology, Pusan National University School of Medicine and Pusan National University Hospital Biomedical Research Institute, 179 Gudeok-ro, Seo-gu, Busan 49241, Korea.

Tel: +82-51-240-7347, Fax: +82-51-247-5443, E-mail: joon501@naver.com 
The mechanism by which varicocele causes scrotal pain is not clearly known, but the following mechanisms have been proposed [2,3]: compression of the surrounding neural fibers by the dilated venous complex, increased scrotal temperature, oxidative stress in the testicular parenchyma, and tissue ischemia secondary to venous stasis. Varicocele is relatively easy to diagnose. The therapeutic effect of varicocele repair, also commonly known as varicocelectomy, on male infertility is well known. However, practitioners who treat patients with varicocele complaining of scrotal pain should take care when choosing a treatment method and counseling patients. This is because patients' satisfaction with varicocelectomy in terms of scrotal pain is not as well established as the effects of varicocelectomy on male infertility [4]. When treating painful varicocele, conservative management may be initially provided, including limitations of physical activities, scrotal elevation, and treatment with nonsteroidal antiinflammatory analgesics. However, these approaches often do not contribute to pain management [4]. Varicocelectomy is an alternative treatment for patients with painful varicocele who do not adequately respond to conservative management [2]. However, the success rate of varicocelectomy for painful varicocele has been reported to vary considerably across studies [4,5]. Symptom improvement has been reported in up to $100 \%$ of patients after surgical repair of a painful varicocele [6]. In contrast, a relatively low improvement rate of $61 \%$ has also been reported [7]. These discrepancies in the reported surgical success rate in the treatment of painful varicocele and the lack of predictive parameters for surgical outcomes make it difficult for urologists to treat patients with painful varicocele. With this background, this study aimed to evaluate the relationships of varicocele characteristics and surgical methods with the pain resolution rate post-varicocelectomy.

\section{MATERIALS AND METHODS}

\section{Literature search}

This meta-analysis was performed according to the Preferred Reporting for Systematic Reviews and Metaanalysis Protocols (PRISMA-P) Statement [8]. The literature search was conducted independently by two authors (P.H.J. \& P.J.H.) using PubMed (MEDLINE) and Embase. The keywords used for the search were "varicocele," "varicocelectomy OR ligation OR repair," and "pain OR painful." The search was limited to studies published in the English language between 1980 and December 2018.

\section{Selection criteria}

Two authors (P.J.H. \& P.H.J.) independently reviewed the titles and abstracts of the related articles. Studies were evaluated for eligibility, and those fulfilling the following inclusion criteria and none of the exclusion criteria were selected for full-text review. Any discrepancies regarding the inclusion or exclusion of a study were discussed, and any disagreements between reviewers were resolved by consensus. For this analysis, we included studies on pain resolution in patients who underwent surgical ligation or clipping for painful varicocele. Only studies presenting a comparison of the postoperative pain resolution rate (complete or partial resolution) according to at least one of following factors were considered in our analysis: varicocele grade, pain quality, and surgical approach or technique. Postoperative pain must have been evaluated after at least 1 month of follow-up. The following exclusion criteria were used: (1) the use of percutaneous occlusion by an intravenous injection of various materials to occlude the varicocele, including retrograde percutaneous embolization or antegrade sclerotherapy; (2) recurrent varicocele or secondary varicocele caused by other diseases, such as nutcracker syndrome, thrombosis, or a retroperitoneal tumor; and (3) pain due to other causes, including testicular torsion, epididymitis or orchitis, prostatitis, and testicular trauma.

\section{Statistical methods}

We performed all comparative analyses with $R$ software (version 3.5.3) using the packages meta and metafor. The extracted data on pain resolution for each parameter were entered and the results are expressed as an odds ratio (OR) and 95\% confidence interval (CI) for dichotomous outcomes. Quantified heterogeneity between studies was reported using Higgins' $\mathrm{I}^{2}$, and, for the random-effects model, $\tau^{2}$. An $\mathrm{I}^{2}$ value over $50 \%$ was considered to indicate significant heterogeneity. The weighting method used was a fixed-effect model if there was no significant heterogeneity between studies, or a random-effects model if significant heterogeneity was observed. Due to the small number of studies included in this analysis, an assessment of publication bias was not conducted. Statistical significance was set at a $p<0.05$. 


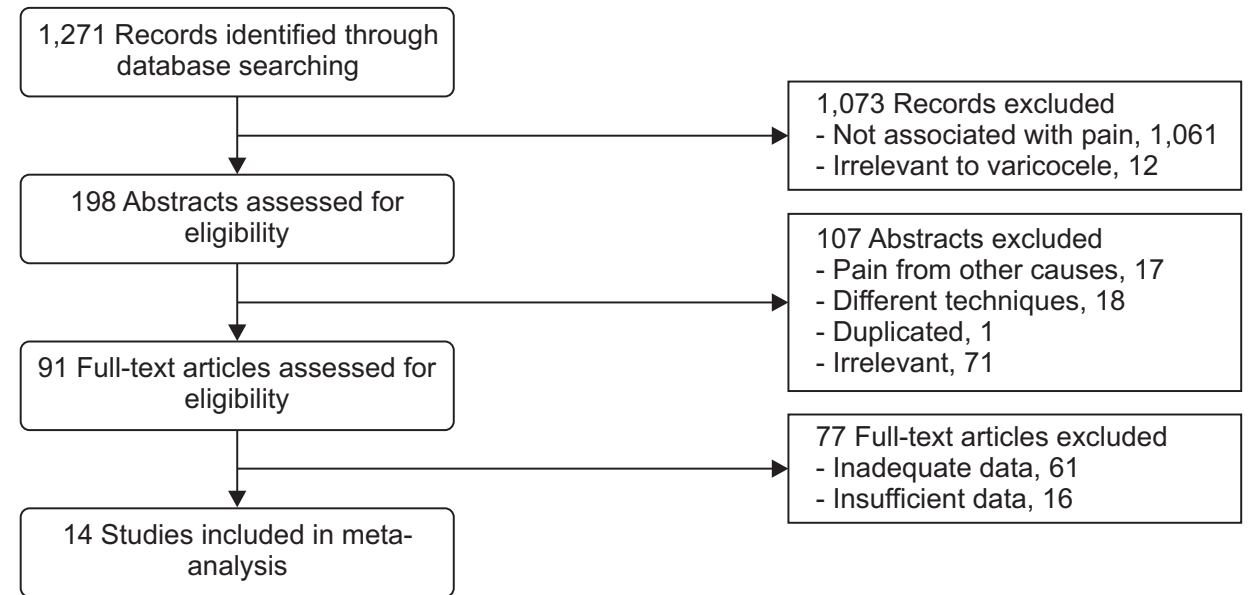

\section{Ethics statement}

This meta-analysis does not involve human subjects and did not require IRB review.

\section{RESULTS}

A total of 1,271 studies were identified through electronic and manual reference searches. Of these, 1,061 studies were not associated with pain, and 12 were considered irrelevant after review of the titles and abstracts. Of the 198 remaining studies, 91 met the inclusion and exclusion criteria after full-text assessment and were further analyzed for potential bias. At the conclusion of the evaluation, 14 studies comprising 1 randomized controlled trial, 4 prospective studies, and 9 retrospective studies were included in the metaanalysis (Fig. 1).

\section{Varicocele grade}

Nine of the 14 studies focused on the correlation between varicocele grade and surgical outcome; in these studies, varicocele grade I was reported in 45 patients; grade II in 269 patients; and grade III in 422 patients. There were no significant differences in the postoperative pain resolution rate with respect to varicocele grades I, II, and III ( $p>0.05)$, suggesting that pain resolution was not correlated with varicocele grade (Fig. 2).

\section{Pain quality}

Eight studies focused on the correlation between pain quality and surgical outcomes. These studies included 192 patients with dull pain, 157 patients with pulling or dragging pain, 93 patients with throbbing pain, 113 patients with sharp pain. The resolution rate
Fig. 1. Flow diagram of the identification and selection of the studies. for dull pain was significantly higher than that for dragging pain (OR, 5.73; 95\% CI, 1.78-18.41; $\mathrm{p}=0.003$ ) or sharp pain (OR, 8.72; 95\% CI, 2.91-26.08; $\mathrm{p}=0.0001$ ), while there were no statistically significant differences in the pain resolution rate between other qualities of pain. The results showed that pain quality was associated with the pain resolution rate and that the pain resolution rate was significantly higher in varicocele patients with dull pain than in those with dragging or sharp pain (Fig. 3).

\section{Surgical approach}

Ten studies were eligible for a comparison of the efficacy of different surgical approaches for pain resolution, with 338 patients undergoing high ligation, 388 undergoing inguinal varicocelectomy, and 460 undergoing subinguinal varicocelectomy. Subinguinal ligation was significantly more effective in terms of pain resolution than high ligation (OR, 2.73; 95\% CI, 1.54-4.85; $\mathrm{p}=0.0006$ ). There were no significant differences between the other surgical approaches in terms of the likelihood of postoperative pain resolution (Fig. 4).

\section{Surgical technique}

Three studies that met the inclusion criteria presented data relevant for an evaluation of the effect of different surgical techniques on the pain resolution rate, including 151 patients undergoing laparoscopic varicocelectomy and 276 undergoing microsurgery. The pain resolution rate was significantly higher after microsurgical varicocelectomy than after laparoscopic varicocelectomy (OR, 7.12; 95\% CI, 3.78-13.44; $\mathrm{p}<0.0001)$ (Fig. 4). 


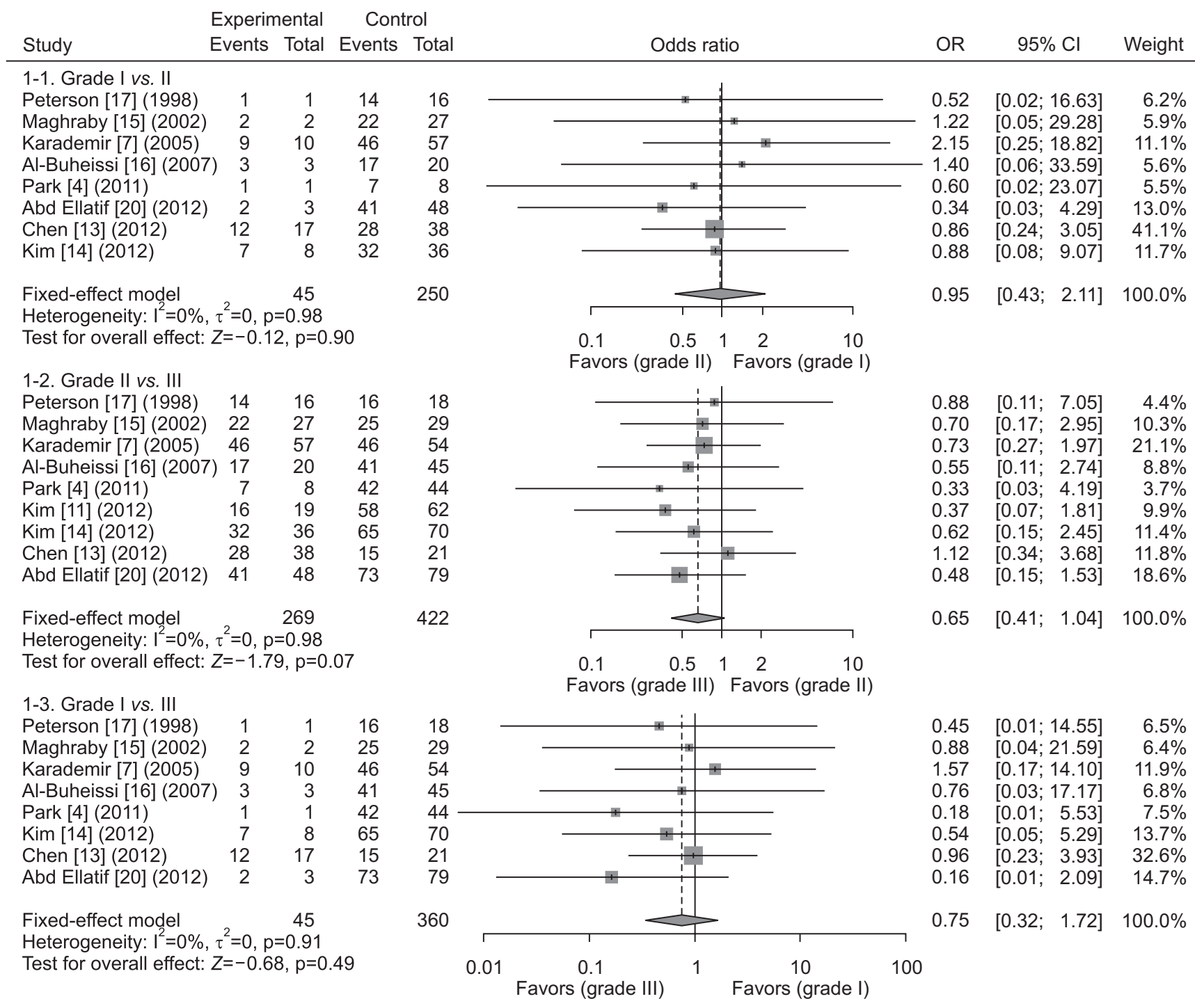

Fig. 2. Pooled results of pain resolution according to varicocele grade.

\section{DISCUSSION}

Chronic scrotal pain remains a challenging urological problem for practitioners to manage. It is a frustrating symptom since it is difficult to determine the cause and to treat it effectively. There are still no universally accepted treatment guidelines [1]. Varicocele is a cause of scrotal pain, and it is recommended to perform conservative treatment initially. If there is no response or if conservative treatment is ineffective, surgical repair (i.e., varicocelectomy) can be performed [3]. Contrary to what may be expected theoretically, there is no definitive opinion as to whether varicocelectomy can adequately treat scrotal pain in real-world practice settings. Therefore, studies on the effects of varico- celectomy in the treatment of painful varicocele continue to be conducted [9]. However, for each study, the indicators used in the analysis are different, and the numbers of patients corresponding to each indicator are unequal [10]. From the perspective of research design, high-quality research is rare because it is difficult to conduct research into this issue with a prospective design as a randomized controlled trial. Therefore, it is important to investigate the tendencies observed in previous studies through a meta-analysis.

In the present study, no significant association was observed between the preoperative varicocele grade and postoperative pain resolution. In this regard, our study is consistent with the meta-analysis of Han et al [10]. Although there was no statistically significant 


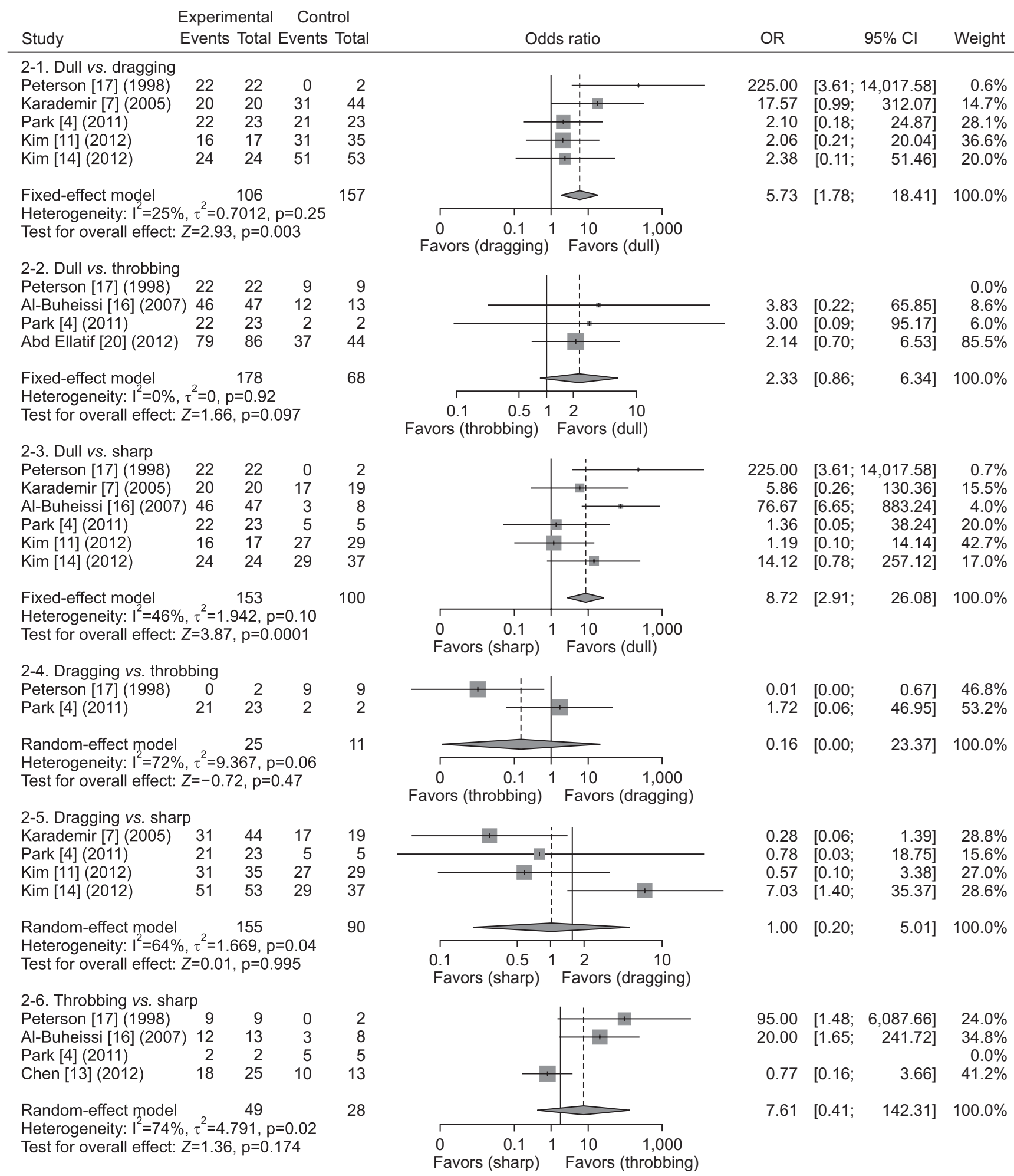

Fig. 3. Pooled results of pain resolution according to the type of pain.

difference in the pain resolution rate between patients with varicocele grades II and III, the pain resolution rate was higher in patients with grade III varicocele [11]. This is presumed to be due to the greater improvement in pain postoperatively, since patients with varicocele grade III experience severe preoperative pain [3]. Yaman et al [12] also reported that varicocele grade was associated with the likelihood of postoperative pain 


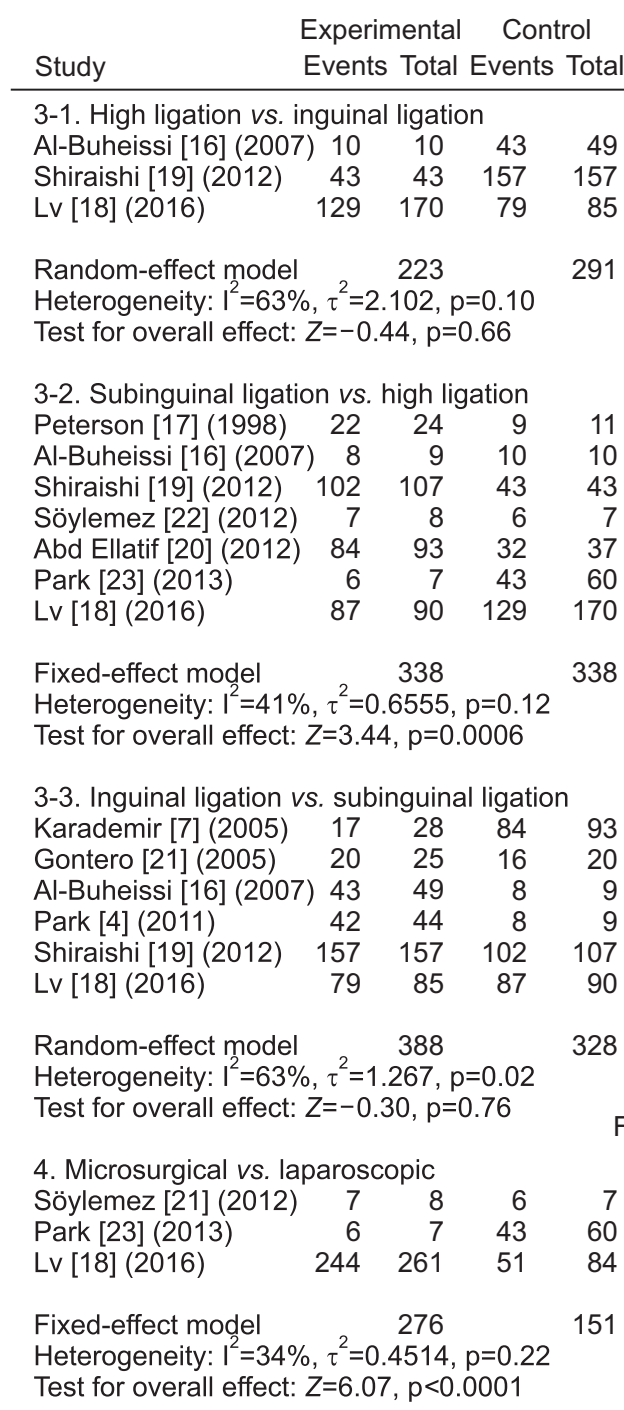

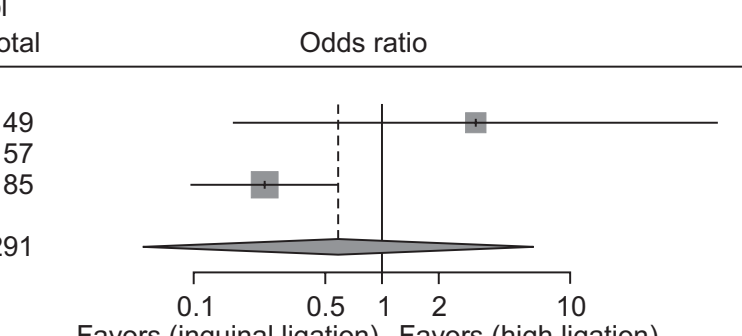

Favors (inguinal ligation) Favors (high ligation)

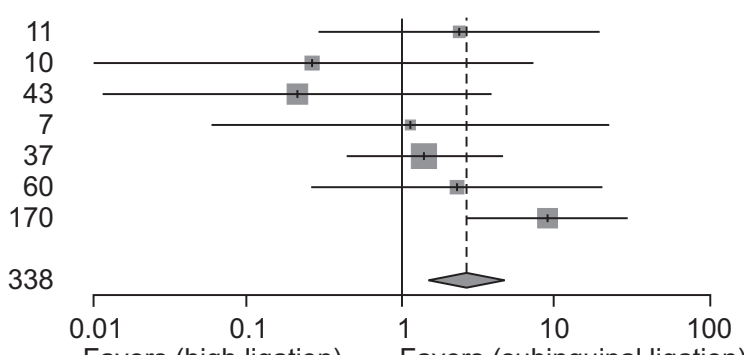

$2.44 \quad[0.30 ; 20.12] \quad 6.8 \%$

$0.27 \quad[0.01 ; 7.51] \quad 9.9 \%$

$0.21 \quad[0.01 ; 3.96] \quad 20.8 \%$

$1.17 \quad[0.06 ; 22.94] \quad 5.3 \%$

$1.46 \quad[0.45 ; \quad 4.68] \quad 29.2 \%$

$2.37 \quad[0.27 ; 21.20] \quad 8.5 \%$

$9.22 \quad[2.77 ; 30.71] \quad 19.6 \%$

$2.73 \quad[1.54 ; 4.85] 100.0 \%$

$\begin{array}{rrrr}3.14 & {[0.16 ;} & 60.21] & 34.6 \% \\ & & 0.0 \% \\ 0.24 & {[0.10 ;} & 0.59] & 65.4 \% \\ & & & \\ 0.58 & {[0.05 ;} & 6.49] & 100.0 \%\end{array}$

Favors (high ligation) Favors (subinguinal ligation)

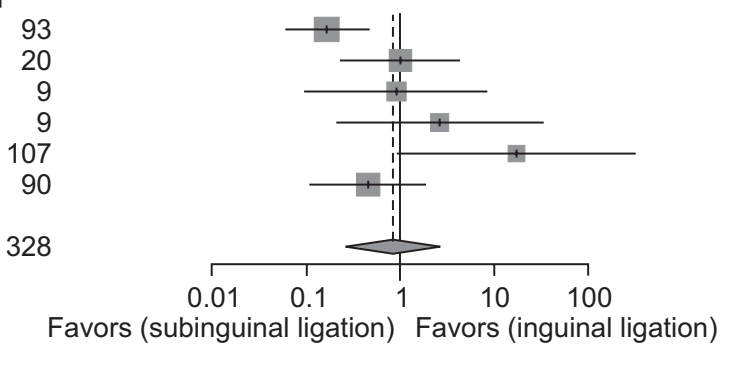

$\begin{array}{rlrr}0.17 & {[0.06 ;} & 0.46] & 23.4 \% \\ 1.00 & {[0.23 ;} & 4.35] & 19.7 \% \\ 0.90 & {[0.09 ;} & 8.48] & 14.0 \% \\ 2.62 & {[0.21 ;} & 32.52] & 12.4 \% \\ 16.90 & {[0.92 ;} & 308.95] & 10.4 \% \\ 0.45 & {[0.11 ;} & 1.88] & 20.1 \% \\ & & & \\ 0.83 & {[0.26 ;} & 2.71] & 100.0 \%\end{array}$

\begin{tabular}{l|l|lllll}
7 & 1 & 1.17 & {$[0.06 ;$} & $22.94]$ & $11.3 \%$
\end{tabular} 60 84 51

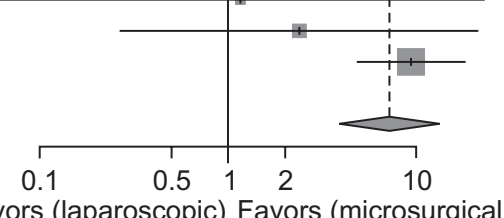

$2.37 \quad[0.27 ; 21.20] \quad 18.1 \%$

$9.29 \quad[4.81 ; 17.94] \quad 70.7 \%$

$7.12[3.78 ; 13.44] 100.0 \%$

Fig. 4. Pooled results of pain resolution according to the method used to treat varicocele.

resolution. In contrast, we found that the preoperative varicocele grade was not associated with pain resolution based on univariate and multivariate analyses in our previous study [4].

Most studies evaluating pain resolution after varicocelectomy have the limitations of an unequal number of patients between varicocele grades. The number of patients with grade I varicocele was significantly smaller than the number of patients with grade II or III varicocele. In the studies included in the present analysis, except for the studies by Chen [13] and Karademir et al [7], the number of patients with grade I varicocele was less than 10 . To elucidate the relationship between varicocele grade and the effect of varicocelectomy on pain resolution, more studies with a comparable number of patients across all varicocele grades are required.

A thorough understanding of the characteristics of pain induced by varicocele is helpful in establishing a treatment strategy and counseling patients [14]. Pain usually occurs intermittently, and its quality varies. The quality of pain can be classified as dull, dragging, throbbing, or sharp [4]. In this study, patients with dull pain showed a significantly higher likelihood of pain resolution than those with dragging or sharp pain. There was no significant difference between other qualities of pain. The results of our study are largely consistent with those of previous studies that have analyzed pain quality as a comparative factor [4]. Although one study grouped dull and dragging pain [13], and another study grouped dull and throbbing pain in the same category [15], most studies have shown 
a higher likelihood of pain resolution after surgery in varicocele patients complaining of preoperative dull pain than in those with other qualities of pain $[7,14,16,17]$. In line with these studies, some reviews and meta-analysis reported that the postoperative pain resolution rate was significantly higher when dull pain was experienced preoperatively [3,9,13]. The reason for the higher likelihood of postoperative pain resolution in patients with dull pain remains unclear. The classic pain of varicocele appears to be a "congestive" or "dull" pattern; this may be related to the mechanism of varicocele, which involves increased hydrostatic pressure in the valveless vein of the pampiniform plexus [17]. Sharp pain is considered to be a form of non-classical pain in patients with varicocele. Therefore, for patients with dull pain, the mechanism underlying the occurrence of pain is directly related to varicocele, but the relationship between the occurrence of sharp pain and varicocele may be weaker. It can be assumed that sharp pain is induced by another cause or by a complex combination of causes. For this reason, it is possible that the postoperative improvement in dull pain can be expected to be greater. However, further research is needed to verify this hypothesis.

To evaluate the difference in the pain resolution rate according to surgical methods, the surgical approach was classified as high, inguinal, or subinguinal, and the surgical technique was classified as laparoscopic varicocelectomy or microsurgical varicocelectomy.

Except for the studies of Lv et al [18] and Shiraishi et al [19], most studies reported no significant difference in the pain resolution rate between surgical approaches [3,4,16,20,21]. Shiraishi et al [19] showed that subinguinal ligation was significantly more effective in terms of pain resolution than inguinal ligation, and $\mathrm{Lv}$ et al [18] reported that subinguinal ligation yielded a significantly higher pain resolution rate than inguinal or high ligation. In our study, subinguinal ligation showed a significantly higher pain resolution rate than high ligation, but not inguinal ligation. The present study is inconsistent with the meta-analysis of Han et al [10], in which subinguinal ligation was associated with a higher pain resolution rate than inguinal ligation. The prospective study of Abd Ellatif et al [20] compared high and subinguinal ligation, but in the meta-analysis performed by Han et al [10], the data from the study of Abd Ellatif et al [20] were entered into the comparison between inguinal and subinguinal ligation. Another study reported distinct results from ours. Karademir et al [7] reported that external spermatic vein ligation was a significant factor related to pain resolution regardless of the surgical approach (inguinal or subinguinal).

A significant association was found between the surgical technique and pain resolution in our study. Microsurgical varicocelectomy showed a statistically significantly higher pain resolution rate than laparoscopic varicocelectomy. Unfortunately, only 3 studies of this issue were eligible for inclusion in the present meta-analysis. Furthermore, the number of patients who underwent each surgical procedure was uneven across these studies [22,23]. Further studies on the effect of varicocelectomy on pain resolution according to the surgical technique are needed.

In this meta-analysis, varicocele grade, pain quality, and surgical approach and technique were selected as indices for comparison. However, in studies on the pain resolution rate after varicocelectomy, various other indicators, such as age, history of conservative treatment, varicocele location, pain duration, testis volume difference, and body mass index (BMI), were considered to be useful parameters [3,4]. Moreover, some studies reported that the longer the duration of pain preoperatively, the greater was the likelihood of pain resolution after varicocelectomy [14,24]. In another study, poor outcomes were reported for patients with varicocele who had a $\mathrm{BMI}>22 \mathrm{~kg} / \mathrm{m}^{2}$ [4].

There are several important limitations of this study. First, the sample size for each indicator evaluated was unequal in the studies included in our meta-analysis. In particular, the number of patients with grade I varicocele was extremely small. Second, we used only varicocele grade, pain quality, and surgical approach and technique as indicators, and did not include all factors that could potentially affect the pain resolution rate. Third, the definition of pain quality and pain resolution is subjective and subject to the sensitivity of each patient; furthermore, it differs from study to study, making it difficult to perform simple comparisons. Standardized definitions of pain quality and pain intensity are needed. Lastly, most studies had a retrospective design. Well-designed studies, such as prospective and randomized controlled trials, were lacking. 


\section{CONCLUSIONS}

In this study, patients with varicocele complaining of dull pain preoperatively were more likely to experience postoperative pain resolution than those complaining of dragging or sharp pain. We also found that subinguinal ligation was more effective in terms of pain resolution than high ligation, and that microsurgical varicocelectomy showed better surgical outcomes than laparoscopic varicocelectomy. However, there were no significant differences in the pain resolution rate according to varicocele grade.

\section{Conflict of Interest}

The authors have nothing to disclose.

\section{Author Contributions}

Conceptualization: JHP, HJP. Data curation: JHP, KP, HJP. Formal analysis: JHP, KP. Funding acquisition: HJP. Investigation: JHP, HJP. Methodology: JHP, KP, HJP. Project administration: JHP, HJP. Resources: JHP, HJP. Software: JHP, HJP. Supervision: NCP, JHP. Validation: HJP. Visualization: JHP. Writing - original draft: JHP, HJP. Writing - review \& editing: JHP, HJP.

\section{REFERENCES}

1. Tan WP, Levine LA. What can we do for chronic scrotal content pain? World J Mens Health 2017;35:146-55.

2. Owen RC, McCormick BJ, Figler BD, Coward RM. A review of varicocele repair for pain. Transl Androl Urol 2017;6:S209.

3. Paick S, Choi WS. Varicocele and testicular pain: a review. World J Mens Health 2019;37:4-11.

4. Park HJ, Lee SS, Park NC. Predictors of pain resolution after varicocelectomy for painful varicocele. Asian J Androl 2011; 13:754-8.

5. Lundy SD, Sabanegh ES Jr. Varicocele management for infertility and pain: a systematic review. Arab J Urol 2017;16:15770.

6. Elzanaty S, Johansen CE. Effect of microsurgical subinguinal varicocele repair on chronic dull scrotal pain in men with grade II-III lesions. Curr Urol 2017;9:188-91.

7. Karademir K, Senkul T, Baykal K, Ateş F, Işeri C, Erden D. Evaluation of the role of varicocelectomy including external spermatic vein ligation in patients with scrotal pain. Int J Urol
2005; $12: 484-8$.

8. Moher D, Shamseer L, Clarke M, Ghersi D, Liberati A, Petticrew $\mathrm{M}$, et al. Preferred reporting items for systematic review and meta-analysis protocols (PRISMA-P) 2015 statement. Syst Rev 2015;4:1.

9. Abrol N, Panda A, Kekre NS. Painful varicoceles: role of varicocelectomy. Indian J Urol 2014;30:369-73.

10. Han DY, Yang QY, Chen X, Ouyang B, Yao B, Liu GH, et al. Who will benefit from surgical repair for painful varicocele: a meta-analysis. Int Urol Nephrol 2016;48:1071-8.

11. Kim SO, Jung H, Park K. Outcomes of microsurgical subinguinal varicocelectomy for painful varicoceles. J Androl 2012;33:872-5

12. Yaman O, Ozdiler E, Anafarta K, Gögüus O. Effect of microsurgical subinguinal varicocele ligation to treat pain. Urology 2000;55:107-8.

13. Chen SS. Factors predicting symptomatic relief by varicocelectomy in patients with normospermia and painful varicocele nonresponsive to conservative treatment. Urology 2012; 80:585-9.

14. Kim HT, Song PH, Moon KH. Microsurgical ligation for painful varicocele: effectiveness and predictors of pain resolution. Yonsei Med J 2012;53:145-50.

15. Maghraby HA. Laparoscopic varicocelectomy for painful varicoceles: merits and outcomes. J Endourol 2002;16:107-10.

16. Al-Buheissi SZ, Patel HR, Wazait HD, Miller RA, Nathan S. Predictors of success in surgical ligation of painful varicocele. Urol Int 2007;79:33-6.

17. Peterson AC, Lance RS, Ruiz HE. Outcomes of varicocele ligation done for pain. J Urol 1998;159:1565-7.

18. Lv JX, Wang LL, Wei XD, Zhang Z, Zheng TL, Huang YH, et al. Comparison of treatment outcomes of different spermatic vein ligation procedures in varicocele treatment. Am J Ther 2016;23:e1329-34.

19. Shiraishi K, Oka S, Ito H, Matsuyama H. Comparison of the results and complications of retroperitoneal, microsurgical subinguinal, and high inguinal approaches in the treatment of varicoceles. J Androl 2012;33:1387-93.

20. Abd Ellatif ME, Asker W, Abbas A, Negm A, Al-Katary M, ElKaffas $\mathrm{H}$, et al. Varicocelectomy to treat pain, and predictors of success: a prospective study. Curr Urol 2012;6:33-6.

21. Gontero P, Pretti G, Fontana F, Zitella A, Marchioro G, Frea B. Inguinal versus subinguinal varicocele vein ligation using magnifying loupe under local anesthesia: which technique is preferable in clinical practice? Urology 2005;66:1075-9.

22. Söylemez H, Penbegül N, Atar M, Bozkurt Y, Sancaktutar AA, Altunoluk B. Comparison of laparoscopic and microscopic subinguinal varicocelectomy in terms of postoperative scrotal 


\section{MEN's HEALTH}

pain. JSLS 2012;16:212-7.

23. Park YW, Lee JH. Preoperative predictors of varicocelectomy success in the treatment of testicular pain. World J Mens Health 2013;31:58-63
Jih Hoon Park, et al: Varicocelectomy for Painful Varicocele

24. Altunoluk B, Soylemez H, Efe E, Malkoc O. Duration of preoperative scrotal pain may predict the success of microsurgical varicocelectomy. Int Braz J Urol 2010;36:55-9. 